\title{
The influence of the monomer shape in the first stage of dust growth in the protoplanetary disk
}

\author{
I. Bertini, P. J. Gutierrez, and W. Sabolo
}

\begin{abstract}
Instituto de Astrofísica de Andalucía, CSIC, Camino Bajo de Huétor 50, 18008 Granada, Spain
\end{abstract}
e-mail: [bertini;pedroj;wsabolo]@iaa.es

Received 1 April 2009 / Accepted 6 July 2009

ABSTRACT

\begin{abstract}
Context. Laboratory experiments have shown that the initial stage of the dust growth in the protoplanetary disk starts with low-velocity hit-and-stick collisions between sub-micron grains, before the relative impact energy becomes large enough to cause the morphological restructuring of the forming aggregates. The results of these collisions are loose aggregates characterized by fractal dimensions $\leq 2$. Numerical studies generally model this early stage with colliding clusters made of monodisperse spherical monomers.

Aims. In this paper we aimed to investigate how a more complex representation of the monodisperse monomers structure influences the morphology of the final aggregate particles in terms of fractal dimension, porosity, cross-section, and friction time. This study had also the purpose of testing the validity of the current fractal models in representing irregular particles.

Methods. We used three kinds of hit-and-stick aggregation methods: two particle-cluster aggregations producing compact and extremely loose aggregates, respectively, and simplified cluster-cluster aggregation as intermediate class in terms of fluffyness and porosity. In our measures, we used two kinds of monomer shapes: spheres and elongated prolate ellipsoids with axis ratio 3:1 resembling the ones used to model interstellar dust grains.

Results. We found that the monomer shape has little influence $(\$ 2 \%)$ on the fractal dimension of the final aggregate, once large particles are taken into account, independently on the morphological class. Elongated monomers produce structures with larger fractal dimension in the limit of small particles. For loose objects the influence on the porosity is even smaller, whereas elongated monomers give particles with $\sim 5 \%$ higher porosity in the case of large compact fractals. Regardless the ellipsoidal monomer used has, by construction, a cross-section $\sim 26 \%$ larger than the one of the volume-equivalent sphere, the difference in cross-section per unit mass is decreasing when aggregates are considered. Clusters made by elliptical monomers result much coupled to the surrounding gas than objects made by spherical monomers belonging to the same morphological class. Differences in the friction time are estimated to be below $15 \%$. We conclude that the monomer shape influence on the morphology of the final particles during the hit-and-stick aggregation step is much lower than the differences due to considering different morphological classes in modeling and the uncertainties produced in a realistic accretion scenario, where a wide distribution of morphologies is expected. Present models of irregular particles as fractals made by spherical monomers can therefore also represent well more complex shapes, before the restructuring phase is taken into account.
\end{abstract}

Key words. accretion, accretion disks - interplanetary medium - solar system: formation

\section{Introduction}

Laboratory experiments set up to investigate the accretion of dust in the protoplanetary disk, leading to the formation of planets and minor bodies, have shown that the initial stage of dust growth starts with aggregation of sub-micron grains through collisions. The strong coupling with the surrounding disk gas leads to low-velocity hit-and-stick collisions between the grains, driven by brownian motion and differential settling, before the relative impact energy becomes large enough to trigger restructuring mechanisms such as compaction and fragmentation. The sticking is due to contact Van der Waals forces. The results of such hit-and-stick collisions are grains with a fractal structure and fractal dimensions $\leq 2$ (we address the reader to Blum \& Wurm (2008) for an extensive review of these experiments).

Numerical studies usually model this early stage as aggregation of fractals made by monodisperse spherical monomers (Meakin et al. 1989; Dominik \& Tielens 1997; Kempf et al. 1999; Paszun \& Dominik 2006; Ormel et al. 2007; Wada et al. 2007, 2008; Suyama et al. 2008; Zsom \& Dullemond 2008). Such monomers are also used as starting seeds to investigate the further aggregation steps, where dust agglomerates can compact, or fragment, due to the increasing impact relative velocities (Meakin et al. 1989; Dominik \& Tielens 1997; Ormel et al. 2007; Wada et al. 2007, 2008; Suyama et al. 2008; Zsom \& Dullemond 2008).

As underlined in Dominik \& Tielens (1997) and Ormel et al. (2007), the properties of the dust in the early stage are of key importance to the evolution of the planetary disk, because the morphology of the collisional aggregates sets up their subsequent growth, determining the interaction with the surrounding gas. A fractal dimension $\leq 2$ implies that the drag force increases only slowly with the aggregate size and the dust agglomerates couple to the gas much better than more compact structures characterized by a higher fractal dimension.

Every theoretical investigation that increases the level of complexity in treating the shapes of particles in the aggregation context may therefore lead to new insights about the dust accretion process. In that sense interesting results can be retrieved using: a) polydisperse and/or b) non-spherical monomers instead of monodisperse spherical monomers. 
The effects of using a polydisperse collection of spherical monomers in aggregation studies were investigated by several authors (e.g. Ossenkopf 1993; Dominik \& Tielens 1997; Zsom \& Dullemond 2008).

In this paper we address point $b$ ), investigating the effects of different monomer shapes on the three-dimensional structure of the final aggregate in terms of fractal dimension, porosity, cross-section, and friction time. This study allows us to derive hints about the validity of current fractal models in representing the complex morphology of irregular particles. We used three hit-and-stick aggregation methods to take into account different morphological classes of the final aggregates, from extremely compact to extremely loose ones, in order to cover the range of possible fractal dimensions present in nature. Restructuring mechanisms were not considered in the collisions. We called these methods particle-clusteraggregation 1 (PCA1), cluster-cluster-aggregation (CCA), and particle-cluster-aggregation 2 (PCA2). We used convex prolate ellipsoids with axis ratio $(3: 1)$ as monomers and we compared the results with the ones obtained with classical spherical monomers. In Sect. 2 the model of aggregation used is described. In Sect. 3 we show how we measured the fractal mass dimension, the porosity, the cross-section, and the friction time of the final aggregates. In Sect. 4 we analyze and discuss the results.

\section{The model of aggregation}

The PCA1 mechanism is straightforward. The first monomer is placed in the three-dimensional (3D) space with its center of mass coincident with the center of the reference frame. The following monomers, which can be seen as projectiles, are generated randomly over a sphere centered in the center of mass of the growing aggregate (the target), which position is updated after every collision, and they are shot towards the target's center after a random rotation. The size of the generating sphere is determined to be always larger than the target's size in order to avoid additional compaction effects. Once the projectiles collide with the target, they stick to it with a sticking probability equal to unity and the aggregate grows.

The CCA case is slightly more complex. The aggregation mechanism is the same as PCA1 but in this case the projectiles in each aggregation step are formed by clusters of monomers having the same shape of the target. The projectiles are randomly rotated before being set on the collision course towards the center of the target. PCA1 and CCA differ from classical ballistic particle-cluster aggregation (BPCA) and ballistic cluster-cluster aggregation (BCCA) methods (Mukai et al. 1992), respectively, because in PCA1 and CCA the projectile is shot towards the center of mass of the target instead of traveling on a random ballistic course intersecting the target cross section as in BPCA and BCCA. Moreover, the CCA particles are created colliding clusters of the same shape, while original BCCA aggregates were obtained through the collision of clusters with same mass but not identical shape. In the 3D space with monodisperse spherical monomers and a hit-and-stick regime, BPCA particles are characterized by fractal dimension $D_{\mathrm{f}} \approx 3$ and BCCA by $D_{\mathrm{f}} \approx 2$ (Mukai et al. 1992).

The PCA2 mechanism is similar to the PCA1 one, except for the fact that the randomly generated monomers are not forced to move towards the center of mass of the aggregate but they are set to move in the direction of the closest monomer. In this way random chains with extremely elongated loose structures and high porosity are obtained, as described in Bertini et al. (2007). This kind of elongated aggregates morphologically resemble the dust particles obtained in the micro-gravity aggregation experiment CODAG $\left(D_{\mathrm{f}} \approx 1.4\right.$ for aggregates made by monodisperse spherical monomers and having the number of monomers, $N \simeq[10-100]$ ), where the structure of primitive preplanetary dust was investigated (Krause \& Blum 2004).

For the three aggregation methods, the detection of the target-projectile collision is performed, when non-spherical monomers are taken into account, representing the monomers with a grid of points (i.e. as polyhedrons described by a certain number of vertices, properly covering their entire surface) and checking, during the time steps where the distances between the projectile and the target are such that a collision can happen, if one of the projectile's vertices is inside the volume of one of the target's monomers. In this case, the projectile becomes part of the target and the process is repeated. In the case of spherical monomers, the collision detection is made in a simpler way, checking if the distance between the projectile and the closest monomer in the target is lower than the monomer's diameter.

\subsection{The different monomer shapes}

We chose to limit our study to convex monomers, because the collision detection algorithm has been largely successfully tested for this kind of shapes. Moreover, concave shapes are not expected to produce appreciable different results in the hit-andstick regime. They would instead probably lead to different behaviors in the restructuring phase, due to the larger number of surface contacts that concave monomers can exhibit with respect to convex monomers. We used two main monomer shapes. The first one is a simple sphere, in order to compare our results with the particles previously used in the literature. The second one is a polyhedron approximation of an elongated prolate rotation ellipsoid. These monomers have two axis equal and the third one is three times larger than the other two (i.e. they are characterized by an axis ratio (3:1)), resembling the shape of the interstellar grains building up comets, as suggested by Greenberg \& Hage (1990). Both spherical and ellipsoidal monomers have the same volume and the same mass. Examples of the generated PCA1, CCA, and PCA2 particles, with the two different monomer shapes are shown in Fig. 1, where aggregates with $N=500$ are displayed. This monomer number value corresponds to the largest particles analyzed in this work.

Although limited to the case of only two different monomer shapes, we believed this choice was sufficient to provide hints on the influence of the monomer shape on the overall morphology of the final particles since the ellipsoidal shape chosen is extremely elongated and different from a sphere, and can be indeed considered as an extreme case.

\section{The measure of the model's parameters}

The measures of the fractal mass dimension, porosity, crosssection, and friction time of the dust aggregates are described in this section.

\subsection{The fractal mass dimension}

The fractal mass dimension, $D_{\mathrm{f}}$, is a measure of the morphology of an object made of self-similar structures (a fractal), indicating how completely the object fills the space. End-member fractals, 

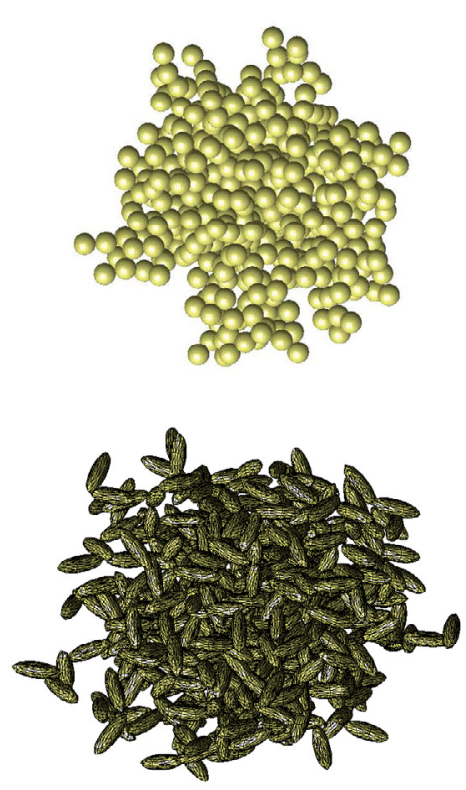
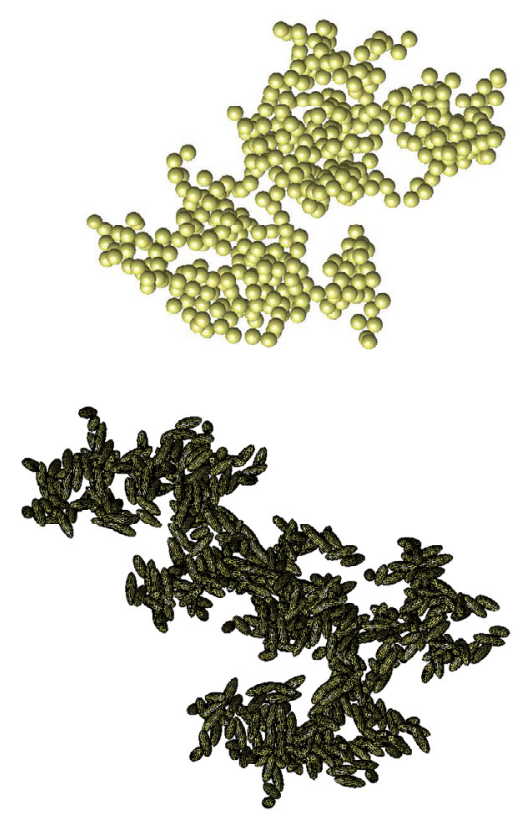
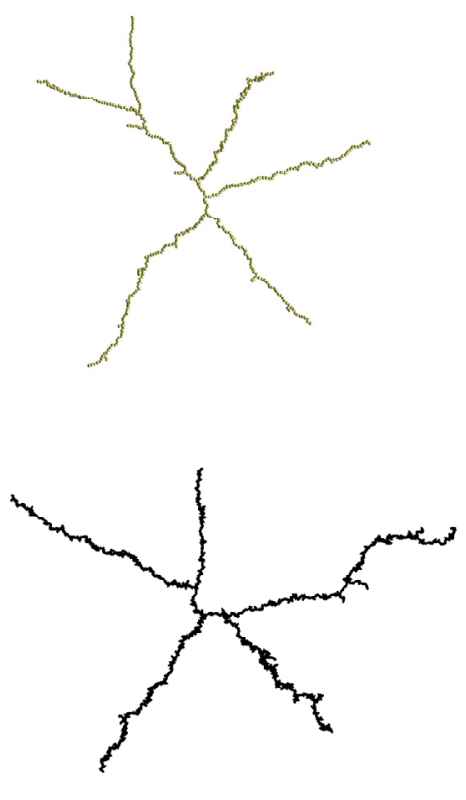

Fig. 1. Examples of aggregate particles obtained with our aggregation codes. We show aggregates with spherical (top) and ellipsoidal 3:1 (bottom) monomers. From left to right PCA1, CCA, and PCA2 particles are displayed, respectively. The number of monomers, $N$, in the aggregates is 500, corresponding to the largest aggregates analyzed in this paper. All the spherical monomers in the figure have the same monomer radius $R_{\mathrm{m}}$ and all the ellipsoidal monomers have the same shape and volume. The views have been scaled individually for clarity.

with $D_{\mathrm{f}} \sim 1$ and $\sim 3$ are characterized by extremely open and compact structures, respectively.

Fractal media obey a power-law relation between their mass and linear size (e.g. Blum \& Wurm 2000):

$m=k R_{\mathrm{g}}^{D_{\mathrm{f}}}$

where $m$ is the mass of the object, $R_{\mathrm{g}}$ is its radius of gyration, measuring the size of the particle, and $k$ is the structural coefficient. Taking then the logarithm of both sides in Eq. (1), we have:

$\log (m)=D_{\mathrm{f}} \log \left(R_{\mathrm{g}}\right)+\log k$

and $D_{\mathrm{f}}$ can be calculated as the slope of the $\log (m)$ versus $\log \left(R_{\mathrm{g}}\right)$ relation. For aggregate particles made by $N$ sub-units, or monomers, of arbitrary shape, with radius of gyration $R_{\mathrm{g}, i}$, $R_{\mathrm{g}}$ can be calculated with the so-called parallel axis theorem (Solvez et al. 1988; Kerfelec et al. 1988; Garcia de la Torre \& Carrasco 2002):

$R_{\mathrm{g}}^{2}=\sum_{i=1}^{N} f_{i}\left(R_{\mathrm{g}, i}^{2}+d_{i}^{2}\right)$

where $f_{i}$ is the mass-fraction of the $i$ th monomer and $d_{i}$ is the distance from its center of mass to the center of mass of the aggregate. In case of monomers having the same density, the mass fraction is the volume fraction, and $f_{i}=V_{i} / \sum_{i=1}^{N} V_{i}$, where $V_{i}$ is the volume of the single monomer. In case of monomers having the same volume, we have $f_{i}=1 / N$. Spherical monomers are characterized by $R_{\mathrm{g}, i}^{2}=\frac{3}{5} R_{\mathrm{m}}^{2}$, where $R_{\mathrm{m}}$ is the monomer radius. For an aggregate made by a monodisperse collection of spherical monomers, Eq. (3) then becomes:

$R_{\mathrm{g}}^{2}=\frac{3}{5} R_{\mathrm{m}}^{2}+\frac{\sum_{i=1}^{N} d_{i}^{2}}{N}$
When dealing with ellipsoidal monomers, we can use an average value for $R_{\mathrm{g}, i}$, following the method used in macromolecular physics by Solvez et al. (1988), Kerfelec et al. (1988), and Garcia de la Torre \& Carrasco (2002). The relative orientation of the monomers, which determines the final structure, influences the $d_{i}$ parameter. As a matter of fact, as underlined in Solvez et al. (1988), Eq. (3) tells us that $R_{\mathrm{g}}$ depends on the dimension of the monomers and the position of their centers, but, if the latter are fixed, $R_{\mathrm{g}}$ does not depend on the monomers orientation. For three-axial ellipsoidal monomers we have $R_{\mathrm{g}, i}^{2}=\frac{a^{2}+b^{2}+c^{2}}{5}$, where $a, b$, and $c$ are the three semi-axis. We are using prolate ellipsoids with $b=c$ and $a=3 b$. Therefore, in our case, $R_{\mathrm{g}, i}^{2}=\frac{11}{5} b^{2}$, and Eq. (3) becomes:

$R_{\mathrm{g}}^{2}=\frac{11}{5} b^{2}+\frac{\sum_{i=1}^{N} d_{i}^{2}}{N}$

Using these equations we were able to calculate $R_{\mathrm{g}}$ for each aggregate, while $N$ was growing at each aggregation step. The final data points in the log-log scale used to calculate $D_{\mathrm{f}}$ for each aggregation method are average points derived from our statistical sample. In the case of spherical monomers the data points are the average of 100 different aggregates. The statistics for ellipsoidal monomers is poorer because the use of the collision detection algorithm increases drastically the necessary computational time. The data points for ellipsoidal monomers are the average of $\sim 50$ different aggregates. Nevertheless, we found that the errors in the determination of the fractal dimension are of the same order of magnitude using spherical monomers with 100 particles and elliptical monomers with $\sim 50$ particles. We conclude therefore that our results have a similar statistical meaning in both cases.

\subsection{The porosity}

A widely-used parameter to describe the effect of volume-filling factor in fractal structures, and irregular particles in general, is 

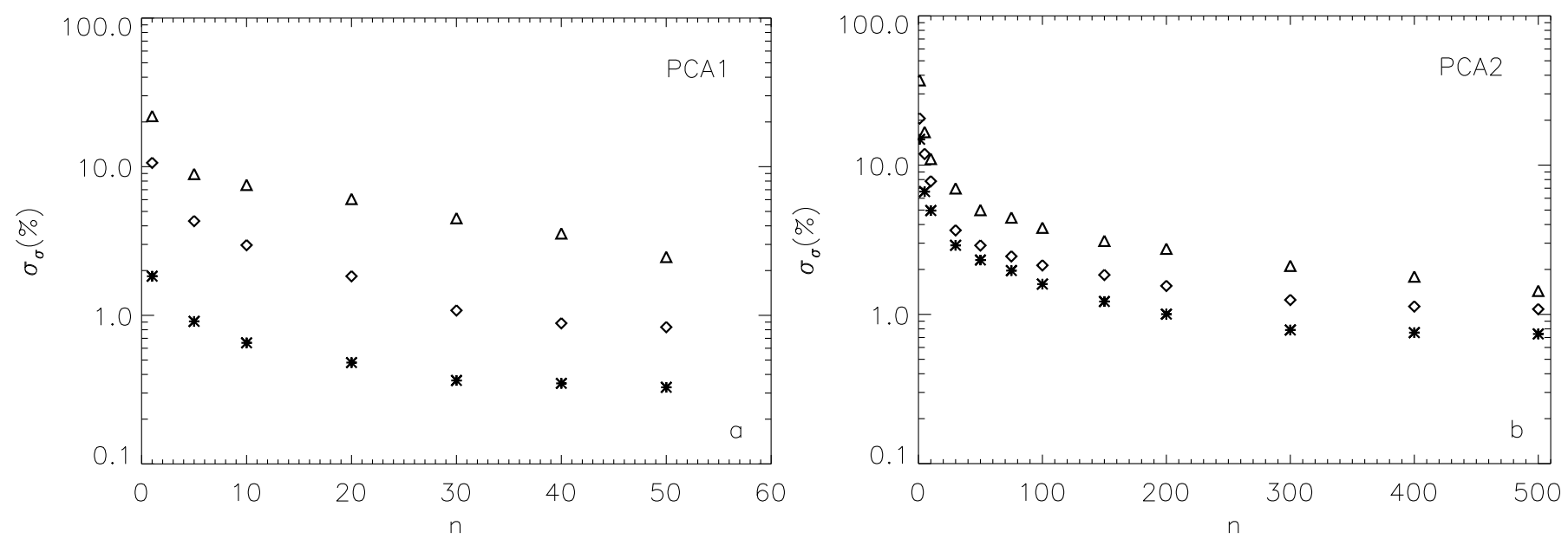

Fig. 2. Percentage standard deviation in the measure of the cross-section, $\sigma_{\sigma}$, versus $n$ for PCA1 a) and PCA2 b) particles. Results are shown for $N=5$ (triangles), $N=50$ (rhombuses), and $N=500$ (stars).

the porosity, $p$, defined as $1-V / V_{\mathrm{t}}$, where $V$ is the volume of the solid part of the particle and $V_{\mathrm{t}}$ is the total volume, generally intended as the volume of the solid part plus the pores (e.g Greenberg \& Hage 1990; Mukai et al. 1992; Kozasa et al. 1992; Davidsson \& Skorov 2002; Petrova et al. 2004; Kimura et al. 2006; Bertini et al. 2007; Das et al. 2008; Voshchinnikov \& Henning 2008). It should be emphasized at this point that the porosity and the fractal dimension need not to be uniquely related. Aggregates with the same porosity may have very different fractal dimensions and vice versa (Leung 1996; Fogel \& Leung 1998). The fractal dimension and the porosity are therefore independent parameters, both characterizing the morphology of fractal aggregates.

In order to calculate the porosity of the aggregates, we applied the method by Mukai et al. (1992), already used by several other authors (e.g Kozasa et al. 1992; Petrova et al. 2004; Kimura et al. 2006; Bertini et al. 2007; Das et al. 2008). Firstly, although fractals do not possess a constant internal density, the so-called characteristic radius, $R_{\mathrm{c}}$, is calculated. $R_{\mathrm{c}}$ represents the radius of a homogeneous sphere having the same radius of gyration of the aggregate and is defined as:

$R_{\mathrm{c}}=\sqrt{\frac{5}{3}} R_{\mathrm{g}}$

The mean porosity, $p$, of an aggregate is then calculated with:

$p=1-\left(\frac{V}{V_{\mathrm{s}}}\right)$

where $V$ is the total volume of the monomers in the aggregate and $V_{\mathrm{s}}$ is the volume of the sphere having radius $R_{\mathrm{c}}$. In case of spherical monomers Eq. (7) becomes:

$p=1-N\left(\frac{R_{\mathrm{m}}}{R_{\mathrm{c}}}\right)^{3}$.

Since we have chosen in our study ellipsoidal and spherical monomers having the same volume, Eq. (8) holds even in the case of ellipsoidal monomers.

\subsection{The cross-section and the friction time}

The cross-section and the friction time are parameters describing the aerodynamical properties of dust particles and the strength of the dust-gas interaction (e.g Kempf et al. 1999). As a matter of fact, the drag force due to the interaction between the gas and the particle embedded in it is proportional to the particle's crosssection and the friction time represents the time that a particle needs to dissipate its kinetic energy of its relative motion to the gas.

In order to calculate the cross-section, $\sigma$, of our aggregates, we used the method described in Meakin \& Donn (1988). This method implies the calculation of the projected area of the aggregate onto the $x y$ plane, $\sigma_{x y}$. If the aggregate contains $N$ monomers, $n N$ random points (where $n=5$ in Meakin \& Donn 1988; and $n=10$ in Meakin et al. 1989) are then selected on the xy plane in a circle of radius $R_{\max }$ enclosing the projected cluster (here $R_{\max }$ is the maximus radius of the cluster measured from its center of mass). If $M$ of these points are in the region containing the projection, an estimate of $\sigma_{x y}$ is:

$\sigma_{x y}=\pi R_{\max }^{2} \frac{M}{n N}$

The procedure is then repeated for the two other perpendicular planes, $x z$ and $y z$, obtaining $\sigma_{x z}$ and $\sigma_{y z}$. The cross section, $\sigma$, is then the average of the measured $\sigma_{x y}, \sigma_{x z}$, and $\sigma_{y z}$.

We found that, using this method, the cross section depends on the number of random points used in the measure of the projected areas. As a matter of fact, the larger the number of random points $n$, the better the $\pi R_{\max }^{2}$ area is filled-up and the smaller the percentage standard deviation, $\sigma_{\sigma}$, in the measure of $\sigma$ is. We estimated the influence of $n$ deriving the $\sigma_{\sigma}$ versus $n$ behavior for single aggregates. We show in Fig. 2 sample relations for PCA1 (Fig. 2a) and PCA2 (Fig. 2b) aggregates made by 5, 50, and 500 spherical monomers, averaging the results over several particles.

It is clear from the plot that the percentage error decreases increasing $n$. Since $R_{\max }$ increases going from compact PCA1 to loose PCA2, once $N$ is fixed, we see that $\sigma_{\sigma}$ increases for the three morphological classes, once $n$ is fixed. Moreover, we found that these behaviors are also $N$-dependant. Smaller aggregates give larger $\sigma_{\sigma}$ than larger aggregates belonging to the same morphological class, once $n$ is fixed. We took into account all these effects when measuring the cross-sections. All the $\sigma$ values were obtained properly choosing the right $n$, according to $N$ and the morphological class, in order to have always $\sigma_{\sigma} \lesssim 1 \%$.

The friction time, $\tau_{\mathrm{f}}$, of a particle embedded in a gas depends on the resistance provided by the gas. For spherical solid grains, 

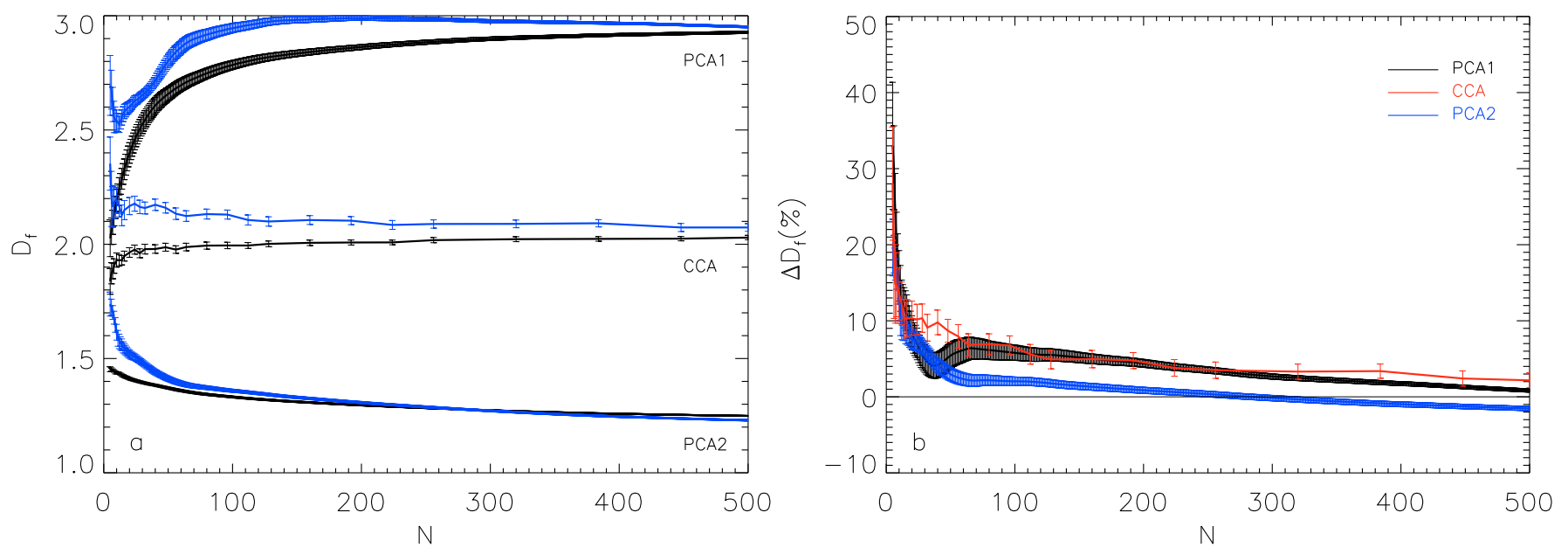

Fig. 3. Fractal dimension $D_{\mathrm{f}}$ a) and percentage fractal dimension difference $\Delta D_{\mathrm{f}} \mathbf{b}$ ) versus the number of monomers in the aggregate, $N$. In a) results are shown for the three aggregation classes with spherical (black lines) and ellipsoidal (blue lines) monomers. In b) the black, red, and blue lines correspond to PCA1, CCA, and PCA2 particles, respectively, and the thin horizontal line marks the zero level. Results are shown for aggregates with $N$ in the range [5-500].

the Epstein formulation (Epstein 1923) holds in a regime characterized by Knudsen numbers (i.e. the ratio between the mean free path length of the gas molecules and the size scale of the solid particle) $K_{n} \gg 1$ :

$\tau_{\mathrm{f}}=\frac{m}{\sigma} \frac{1}{\rho_{\mathrm{g}} v_{\mathrm{th}}}$

where $m$ and $\sigma$ are the mass and the cross-section of the grain, and $\rho_{\mathrm{g}}$ and $v_{\text {th }}$ denote the mass density and the mean thermal velocity of the gas, respectively.

As underlined by Blum et al. (1996) and Kempf et al. (1999), for almost all the astrophysical environments, including the hitand-stick regime we are analyzing, the gas densities are very low and the grain sizes are so small that $K_{n} \gg 1$. Laboratory experiments by Blum et al. (1996) showed that deviation from particle sphericity, using fractal aggregates, causes only minor deviations from the Epstein's law, and that Eq. (10) can be successfully used to calculate $\tau_{\mathrm{f}}$ for irregular particles.

\section{Results analysis}

The results obtained in the measure of the morphology of fractal aggregates are illustrated and discussed in the following section.

\subsection{The fractal mass dimension}

In Fig. 3 a we analyze the $D_{\mathrm{f}}$ versus $N$ behavior, depending on the morphological class and the monomer shape for particles with $N$ in the range [5-500]. The same fractal dimension data points are plotted in Fig. 4a with respect to the $R_{\max }$ over the monomer size parameter, relating in that way our results to real physical sizes. The monomer size is $R_{\mathrm{m}}$ and $b$ for spherical and ellipsoidal monomers, respectively. All data points are shown with correspondent one sigma error bars. CCA aggregates, obtained through the collision of identical clusters, give data points characterized by different series of $N$, according to the number of monomers of the starting seed, $N_{\mathrm{s}}$, used in the first aggregation step. We used starting seeds with $N_{\mathrm{s}}=2,3,5$, and 7 . The data points at $N=500$ were found linearly interpolating the results at $N=448\left(N_{\mathrm{s}}=7\right)$ and $N=512\left(N_{\mathrm{s}}=2\right)$.

It has been shown that the fractal dimension of aggregates changes with their size. Mandelbrot (1992) showed that in particle-cluster aggregation (diffusion-limited in his case) the particles are characterized by fractal dimension increasing with size, tending to an asymptotic value for large clusters, when the growing aggregate is much larger than the added monomer. In CCA particles made by spherical monomers, where two identical clusters take part in each aggregation event, the $D_{\mathrm{f}}$ of the growing aggregate is practically constant at each stage (Kolb et al. 1983). Our results also show that the $D_{\mathrm{f}}$ dependency on $N$ is influenced by the aggregation class. It is clear that compact particles (PCA1) made by spherical monomers have $D_{\mathrm{f}}$ increasing with $N$, appearing to tend to a constant value for large clusters. This behavior is still present in CCA particles but they tend faster than PCA1 to an asymptotic value. We also analyzed for the first time the dependency in extremely fluffy aggregates (PCA2). We found that in this case the fractal dimension is decreasing with increasing $N$, appearing to tend to a constant value for large clusters. Aggregates made by ellipsoidal monomers show a similar trend with $N$ in the PCA 2 case. CCA particles are instead characterized by a gentle decrease of $D_{\mathrm{f}}$ with increasing $N$. PCA1 objects have $D_{\mathrm{f}}$ decreasing in the range $N \sim$ [5-10], then increasing reaching a maximum around $N \sim 200$, and afterwards gently decreasing at larger $N$.

In order to estimate the monomer shape influence on the morphology of the final aggregate, we calculated the percentage difference, $\Delta D_{\mathrm{f}}$, inside a morphological class, between the fractal dimension retrieved from ellipsoidal and spherical monomers. This difference is calculated through the formula:

$\Delta D_{\mathrm{f}}(\%)=100 \frac{\left(D_{\mathrm{fe}}-D_{\mathrm{fs}}\right)}{D_{\mathrm{fs}}}$

where $D_{\mathrm{fs}}$ and $D_{\mathrm{fe}}$ are the fractal dimension obtained from spherical and ellipsoidal monomers, respectively. In Figs. $3 b$ and $4 \mathrm{~b}$ we show the $D_{\mathrm{f}}$ percentage difference versus $N$ and $R_{\max } /$ monomer size using the data plotted in Figs. 3a and $4 \mathrm{a}$, respectively. The three aggregation classes show similar fractal dimension percentage trends with $N . \Delta D_{\mathrm{f}}$ is lowering with increasing $N$. Aggregates made by ellipsoidal monomers show always a fractal dimension higher than correspondent particles made by spherical monomers, except for PCA2 clusters with $N \gtrsim 300$. For particles having $N \gtrsim 20-30$ the difference gets below $10 \%$. The largest objects we analyzed are characterized by an absolute value of $\Delta D_{\mathrm{f}} \lesssim 2 \%$, showing that for large particles 

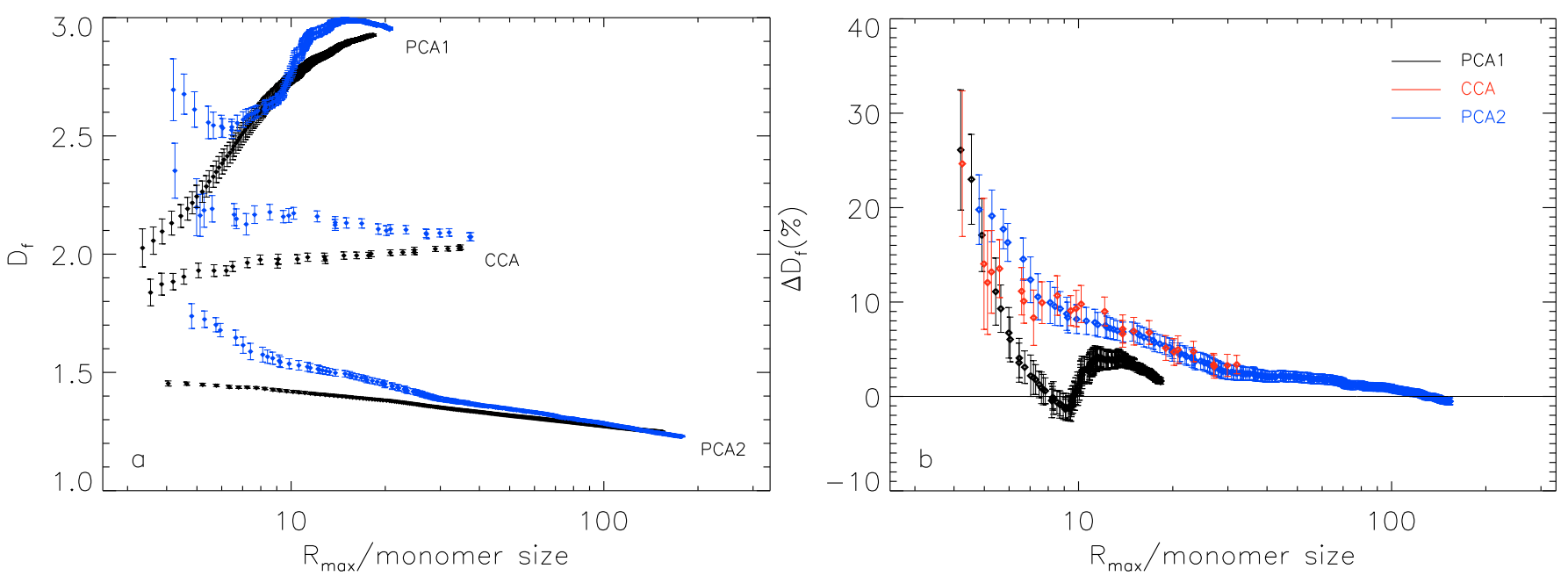

Fig. 4. Fractal dimension $D_{\mathrm{f}}$ a) and percentage fractal dimension difference $\Delta D_{\mathrm{f}} \mathbf{b}$ ) versus the fraction between the maximum dimension of the aggregate $R_{\max }$ and the monomer size. For spherical monomers this fraction is $R_{\max } / R_{\mathrm{m}}$, while for ellipsoidal monomers is $R_{\max } / b$. In a) results are shown for the three aggregation classes with spherical (black lines) and ellipsoidal (blue lines) monomers. In b) the black, red, and blue points correspond to PCA1, CCA, and PCA2 particles, respectively. Results in a) are shown for aggregates with $N$ in the range [5-500], while in b) the size range depends on the common $R_{\max }$ /monomer size interval between results for spherical and ellipsoidal monomers inside a single morphological class. The horizontal thin line in b) marks the zero level.
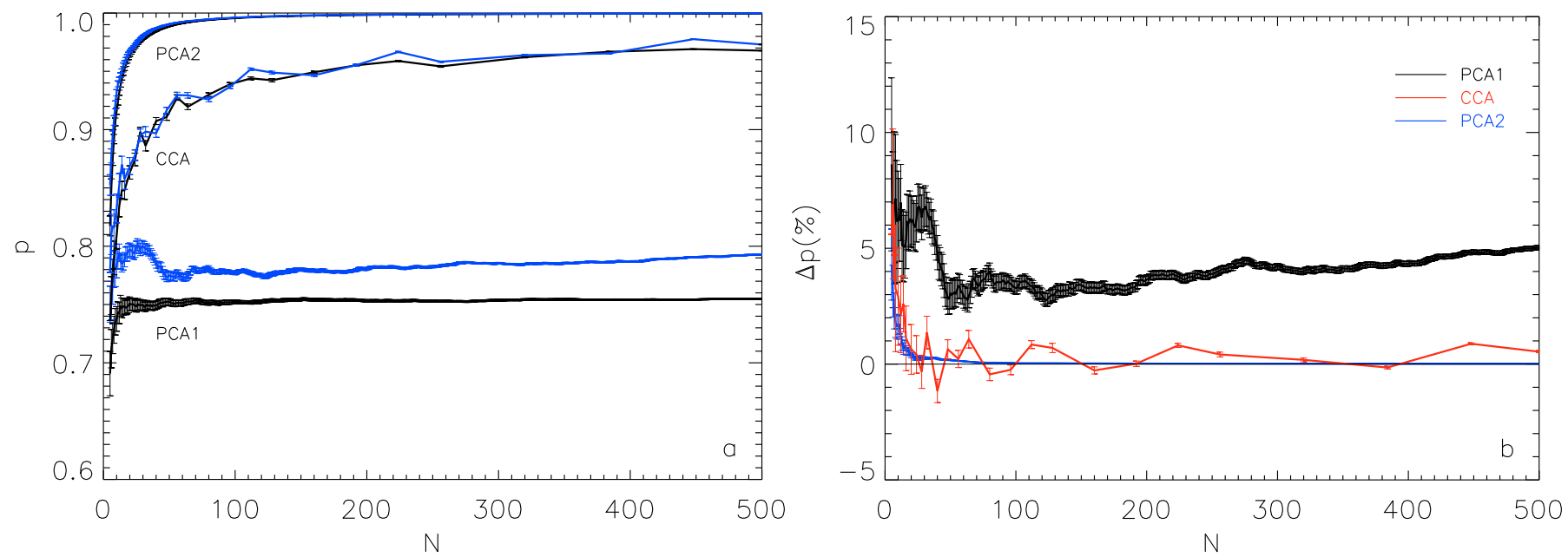

Fig. 5. Porosity $p$ a) and percentage porosity difference $\Delta p$ b) versus the number of monomers in the aggregate, $N$. In a) results are shown for the three aggregation classes with spherical (black lines) and ellipsoidal (blue lines) monomers. In b) the black, red, and blue lines correspond to PCA1, CCA, and PCA2 particles, respectively, and the thin horizontal line marks the zero level. Results are shown for aggregates with $N$ in the range [5-500].

the monomer shape has little influence on the fractal dimension of the final aggregate.

\subsection{The porosity}

In Figs. 5a and 6a we plot the porosity versus the number of monomers $N$ and the parameter $R_{\max } /$ monomer size, respectively. Error bars are given by the standard deviations we found averaging the results in the statistical sample used.

In CCA and PCA2 particles, $p$ increases with $N$, tending to a constant value, although CCA objects show local variations. For PCA2 particles this happens rapidly, while CCAs show a slower growing of the porosity with $N$. Similar fast trend with $N$ is found in PCA1 aggregates made by spherical monomers, whereas corresponding objects made by elliptical monomers show a constant increasing trend of $p$ with $N$, although with local erratic behavior, for $N \gtrsim 50$.

The influence of the monomer shape on the porosity is evaluated in Figs. 5b and 6b, where we show the porosity percentage difference, $\Delta p$, calculated similarly to Eq. (11). CCA and PCA2 structures are characterized by $\Delta p$ decreasing with increasing $N$ and reaching $\Delta p \lesssim 1 \%$ for $N \gtrsim 15-20$, although CCA particles show erratic local variations. We conclude that the monomer shape has extremely little influence in the CCA and PCA 2 cases except for very small particles, where ellipsoidal monomers create systematically more porous structures. The case of compact PCA1 aggregates is somehow different: ellipsoidal monomers produce always more porous structures with a minimum $\Delta p \sim 3 \% . \Delta p$ has a decreasing trend up to $N \simeq 50$ and then increase constantly with $N$, although with local 

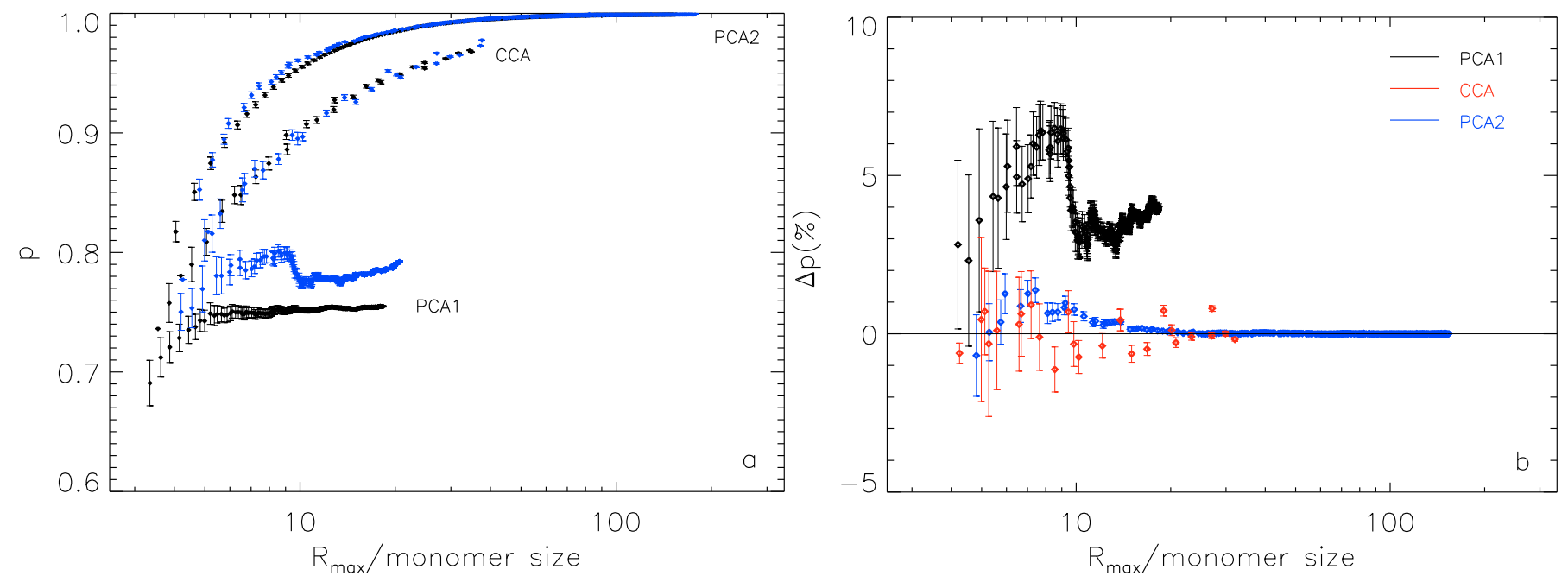

Fig. 6. Porosity $p$ a) and percentage porosity difference $\Delta p$ b) versus the fraction between the maximum dimension of the aggregate $R_{\max }$ and the monomer size. For spherical monomers this fraction is $R_{\max } / R_{\mathrm{m}}$, while for ellipsoidal monomers is $R_{\max } / b$. In a) results are shown for the three aggregation classes with spherical (black lines) and ellipsoidal (blue lines) monomers. In b) the black, red, and blue points correspond to PCA1, CCA, and PCA2 particles, respectively. Results in a) are shown for aggregates with $N$ in the range [5-500], while in b) the size range depends on the common $R_{\max } /$ monomer size interval between results for spherical and ellipsoidal monomers inside a single morphological class. The horizontal thin line in b) marks the zero level.
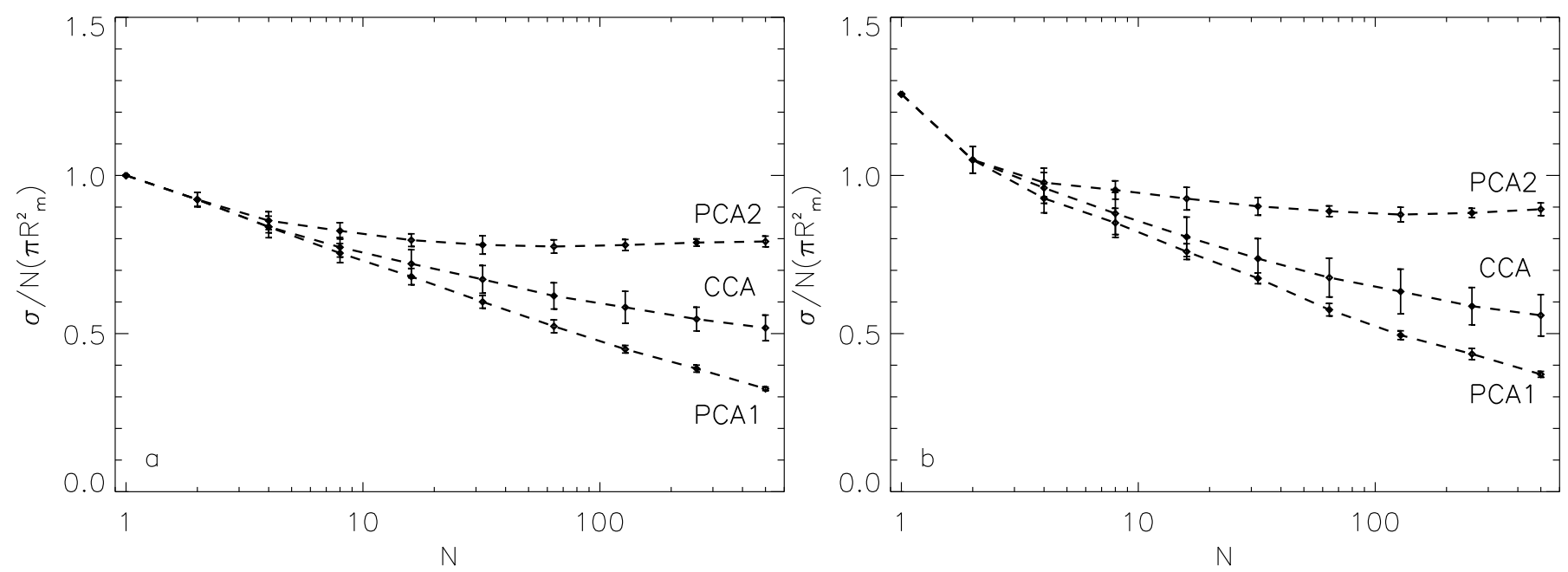

Fig. 7. Cross-section per unit mass, $\sigma / N$, in units of $\pi R_{\mathrm{m}}^{2}$, versus $N$. Results regarding the three morphological classes used are shown for spherical a) and ellipsoidal b) monomers.

variations. $\Delta p$ for the largest particles analyzed with $N=500$ raises up to $5 \%$.

\subsection{The cross-section and the friction time}

The $\sigma / N$ parameter is proportional to the ratio of the gas-drag to the particle mass, or, equivalently, to the inverse of the friction time. It is therefore the dominant factor in establishing the gasgrain interaction and determines, together with the gas velocity field, the motion of the aggregate, as underlined in Meakin \& Donn (1988).

In Fig. 7 we plot the $\sigma / N$ versus $N$ parameter, normalized to the spherical monomer cross-section $\pi R_{\mathrm{m}}^{2}$, for the three morphological classes and the two monomer shapes.

Looking at the results for spherical monomers, a clear trend of the $\sigma / N$ versus $N$ behavior connected with the shape of the aggregates can be found. Loose particles give larger cross sections per unit mass with respect to compact particles when the same $N$ is considered. This implies a larger gas-drag per unit mass and a stronger coupling with the surrounding gas with the consequence that fluffier particles settle more slowly towards the mid-plane of the protoplanetary disk and feel much more the effects of eventual local turbolences. The PCA2 behavior in that sense has to be underlined. While in PCA1 and CCA aggregates $\sigma / N$ decreases increasing $N$, PCA2 objects are characterized by $\sigma / N$ decreasing up to $N \simeq 60$ and then slightly increasing at larger $N$. Nevertheless, considering the error in the measures, $\sigma / N$ is almost constant for $N \gtrsim 10$ in the PCA2 case. This implies that for extremely fluffy aggregates the aerodynamical behavior is practically independent of the particles size. Our results agree with the findings of Meakin \& Donn (1988), Mukai et al. (1992), Ossenkopf (1993), and Minato et al. (2006), for BPCA and BCCA particles, and extend them to extremely fluffy aggregates having $D_{\mathrm{f}}<1.5$.

We found that also aggregates made by ellipsoidal monomers show similar trends, although they always exhibit larger cross-section with respect to fractals made by spherical monomers (see Fig. 7b). This is an expected result, because the 


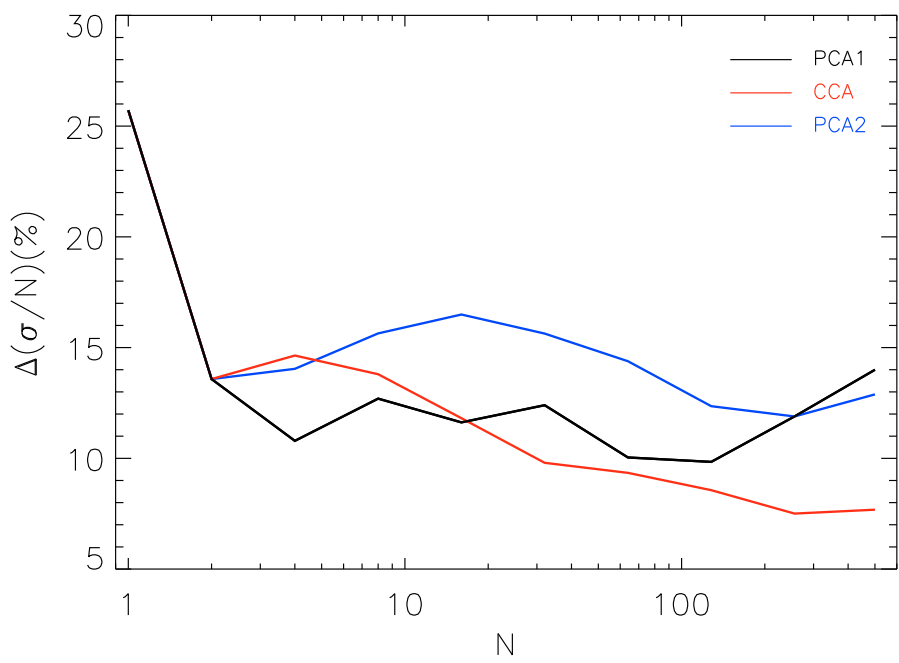

Fig. 8. Percentage cross-section per unit mass $\Delta(\sigma / N)$ difference versus the number of monomers $N$. Black, red, and blue lines stand for PCA1, CCA, and PCA2 aggregates, respectively.

ellipsoids we used in our study have larger cross-section than the volume-equivalent spheres. As a matter of fact, calculating the surface area of the ellipsoidal monomer and applying the Cauchy's theorem (Cauchy 1908), stating that for any convex body the mean value of the projected area is one quarter of its surface area, it follows that the ellipsoidal monomer have a mean cross-section $\sim 26 \%$ larger than the one of the volume-equivalent sphere. It is therefore logical that aggregates made by the ellipsoidal monomers have, by construction, larger cross-section than fractals made by spherical monomers. Nevertheless, looking at Fig. 8, where the percentage difference $\Delta(\sigma / N)$, calculated similarly to Eq. (11), is plotted versus $N$, we can conclude that the difference decreases when aggregates are considered, for the three morphological classes.

Once we consider dust particles in the same position of the protoplanetary disk, the friction time percentage difference, $\Delta \tau_{\mathrm{f}}$, depends only on the inverse of the cross-section per unit mass, because the gas parameters are identical for every object. Using the $\sigma / N$ values previously obtained and Eq. (11), $\Delta \tau_{\text {f }}$ can then be easily derived. We found that clusters of ellipsoidal monomers have lower friction time than correspondent aggregates of spherical monomers. The influence of the monomer shape on the friction time decreases to absolute values $\lesssim 15 \%$, once aggregates are taken into account.

\section{Summary and conclusions}

We analyzed the influence of the monomer shape on the morphology of the final aggregates in terms of fractal mass dimension, porosity, cross-section, and friction time. We used two kinds of monomer shape (spheres and prolate ellipsoids with axis ratio 3:1) and three kinds of aggregation classes, named PCA1, CCA, and PCA2, producing from extremely compact to extremely loose aggregates, respectively.

We found that:

- The value of the fractal mass dimension, $D_{\mathrm{f}}$, calculated with the usual $m \sim R_{\mathrm{g}}^{D_{\mathrm{f}}}$ formula is dependent on the size of the aggregate. The dependency is different for different morphological classes. Compact aggregates show $D_{\mathrm{f}}$ increasing with size, tending to an asymptotic value for larger particles. CCA particles have $D_{\mathrm{f}}$ slightly increasing with size, showing almost constant values. Extremely fluffy aggregates are characterized by fractal dimension slightly decreasing with increasing size and tending to a constant value for large particles. Aggregates made by ellipsoidal monomers show a similar trend with $N$ in the loosest case. PCA1 and CCA particles show different behaviors compared to the correspondent clusters of spherical monomers. Ellipsoidal monomers result in clusters with fractal dimension always larger than correspondent objects made by spherical monomers, except for PCA2 aggregates with $N \gtrsim 300$. The percentage difference in the fractal dimension gets smaller for larger objects in the three aggregation classes. For $N \gtrsim 20-30$ the influence of the monomer shape is estimated to be below $10 \%$ and for the largest particles analyzed gets lower than $2 \%$.

- As a general trend the porosity, calculated as 1-(solid volume of the particle)/(total volume), increases with $N$ in all cases, although with different modalities depending on the morphological class and the monomer shape. Compact particles made by ellipsoidal monomers show this behavior for $N \gtrsim 40$. The monomer shape has little influence on $p$ in the open aggregates CCA and PCA2. The porosity percentage difference is lower than $1 \%$ for $N \gtrsim 15-20$. Smaller particles made by elliptical monomers always exhibit more porous structures with respect to spherical monomers clusters. In compact objects the elongated monomers produce aggregates with higher $p$ and $\Delta p$ is increasing with $N$ for $N \gtrsim 50$, reaching $5 \%$ with $N=500$.

- Loose particles have larger cross-section per unit mass with respect to compact aggregates of the same mass. This implies a stronger coupling with the surrounding gas in the protoplanetary disk. In the extremely fluffy objects the aerodynamical behavior is practically independent of particles size for $N \gtrsim 10$. These trends are not depending on the monomer shape. Although the ellipsoidal monomer used in our measures has an average cross-section $\sim 26 \%$ larger than the one of the volume-equivalent sphere, the difference in the cross-section gets smaller when aggregates are considered. Clusters of ellipsoidal monomers have larger crosssections and lower friction times compared to particles made by spherical monomers. The influence of the monomer shape on the friction time is estimated to be lower than $15 \%$.

Finally, we conclude that the monomer shape has little influence on the morphology of the final aggregate if a hit-and-stick regime is considered. This influence is much lower than the differences induced by using different morphological classes in modeling, dealing with fractal dimension, porosity, and crosssection per unit mass. Moreover, using $N$-particle simulations, Kempf et al. (1999), have shown that in the early stage of dust aggregation the resulting particles exhibit a distribution of morphologies remarkably broad, much larger than the effects of the monomer shape we measured. As a consequence, the present models of irregular dust particles as aggregates of monodisperse spherical monomers, can be also considered representative of more complex shapes, before the restructuring phase of aggregation becomes dominant.

Acknowledgements. I. Bertini and W. Sabolo acknowledge grants by the Junta de Andalucia under contract JA2007-TIC2744. Authors are grateful to the anonymous Referee, who helped improving the quality of the paper with useful comments.

\section{References}

Bertini, I., Thomas, N., \& Barbieri, C. 2007, A\&A, 461, 351

Blum, J., \& Wurm, G. 2000, Icarus, 143, 138

Blum, J., \& Wurm, G. 2008, ARA\&A, 46, 21 
Blum, J., Wurm, G., Kempf, S., \& Henning, T. 1996, Icarus, 124, 441

Cauchy, A. 1908, Memoire sur la rectificacion des courbes et la quadrature des surfaces courbes, presente le 22 Octobre, 1832 (Ire serie), 167

Das, H. S., Das, S. R., Paul, T., Suklabaidya, A., \& Sen, A. K. 2008, MNRAS, 389,787

Davidsson, B. J. R., \& Skorov, Y. V. 2002, Icarus, 156, 223

Dominik, C., \& Tielens, A. G. G. M. 1997, ApJ, 480, 647

Epstein, P. 1923, Phys. Rev., 22, 710

Fogel, M. E., \& Leung, C. M. 1998, ApJ, 501, 175

Garcia de la Torre, J., \& Carrasco, B. 2002, Biopolymers, 63, 163

Greenberg, J. M., \& Hage, J. I. 1990, ApJ, 361, 260

Kempf, S., Pfalzner, S., \& Henning, T. K. 1999, Icarus, 141, 388

Kerfelec, B., Chapus, C., \& Vachette, P. 1988, Eur. Biophys. J.

Kimura, H., Kolokolova, L., \& Mann, I. 2006, A\&A, 449, 1243

Kolb, M., Botet, R., \& Jullien, R. 1983, Phys. Rev. Lett., 51, 1123

Kozasa, T., Blum, J., \& Mukai, T. 1992, A\&A, 263, 423

Krause, M., \& Blum, J. 2004, Phys. Rev. Lett., 93, 021103

Leung, C. M. 1996, Modeling astrophysical phenomena involving dust (NATO Advanced Study Institute on the Cosmic Dust Connection, 3rd Course of the International School of Space Chemistry), 273
Mandelbrot, B. B. 1992, Physica A, 191, 95

Meakin, P., \& Donn, B. 1988, ApJ, 329, L39

Meakin, P., Donn, B., \& Mulholland, G. 1989, Langmuir, 5, 510

Minato, T., Köhler, M., Kimura, H., Mann, I., \& Yamamoto, T. 2006, A\&A, 452, 701

Mukai, T., Ishimoto, H., Kozasa, T., Blum, J., \& Greenberg, J. M. 1992, A\&A, 262,315

Ormel, C. W., Spaans, M., \& Tielens, A. G. G. M. 2007, A\&A, 461, 215

Ossenkopf, V. 1993, A\&A, 280, 617

Paszun, D., \& Dominik, C. 2006, Icarus, 182, 274

Petrova, E. V., Tishkovets, V. P., \& Jockers, K. 2004, Sol. Syst. Res., 38, 309

Solvez, J. A., Iniesta, A., \& Garcia de la Torre, J. 1988, Int. J. Biol. Macromol., 10,39

Suyama, T., Wada, K., \& Tanaka, H. 2008, ApJ, 684, 1310

Voshchinnikov, N. V., \& Henning, T. 2008, A\&A, 483, L9

Wada, K., Tanaka, H., Suyama, T., Kimura, H., \& Yamamoto, T. 2007, ApJ, 661, 320

Wada, K., Tanaka, H., Suyama, T., Kimura, H., \& Yamamoto, T. 2008, ApJ, 677, 1296

Zsom, A., \& Dullemond, C. P. 2008, A\&A, 489, 931 